\title{
Hypotonia and Infantile Spasms: A New Phenotype of Coenzyme $Q_{10}$ Deficiency?
}

\author{
R.J.Huntsman, E.G. Lemire, C.P. Dunham
}

Can. J. Neurol. Sci. 2009; 36: 105-108

Coenzyme $\mathrm{Q}_{10}$ (ubiquinone) deficiency is a rare mitochondrial disorder with less than 45 patients reported worldwide. It is a clinically heterogenous respiratory chain disorder affecting predominantly the central nervous system (CNS) and skeletal muscle ${ }^{1,2}$. In infants, the most common phenotype is the severe infantile multisystemic variant while a phenotype in keeping with Leigh's disease has also been reported ${ }^{1,3,4}$.

While seizures were frequently encountered in patients with Coenzyme $\mathrm{Q}_{10}$ deficiency, none of the patients were reported to have infantile spasms. The most common seizure type is focal seizures associated with focal epileptic discharges on electroencephalogram $(E E G)^{1,4-6}$. While infantile spasms have been reported with various mitochondrial diseases, no patients have been reported to date with infantile spasms secondary to Coenzyme $\mathrm{Q}_{10}$ deficiency ${ }^{7}$.

\section{Case Description}

The patient presented initially at five days-of-age with central hypotonia. Computed tomography scan of the head and EEG were normal. The hypotonia was felt to be secondary to maternal gestational diabetes and the resultant neonatal polycythemia.

She returned to our attention at five months when she developed repetitive jerking movements consisting of forced flexion of the trunk, neck, hips and shoulders. These were most prominent after arousal from sleep and appeared to cluster. On assessment it was evident her neurological status had deteriorated. She was profoundly hypotonic in the trunk and extremities. There was mild weakness of the extremities and tendon reflexes were hypoactive throughout. She appeared to be visually impaired with absent visual fixation and tracking. She was lethargic, awakening only for brief periods of time. She had not met any of her expected developmental milestones such as rolling and raising her chest off the ground when placed prone on her abdomen. She was not bringing her hands together or reaching for objects, neither was she vocalizing or smiling socially. Assessments for abdominal visceromegaly and cardiac function were unremarkable. An EEG showed hypsarrythmia with a burst suppression variant. She was subsequently diagnosed with infantile spasms.

Metabolic workup including serum amino acids, lactate and biotinidase were normal. Measurements of cerebrospinal fluid (CSF) glucose, protein, amino acids and lactate were normal (CSF lactate $1.0 \mathrm{mmol} / \mathrm{l}$ ). Magnetic Resonance Imaging (MRI) showed generalized cerebral atrophy but no definite stigmata of any metabolic disease. Multivoxel Magnetic Resonance
Spectroscopy revealed moderately increased levels of lactate within the brain parenchyma raising the possibility of a mitochondrial disorder.

There was no improvement in the spasms or EEG despite therapy with vigabatrin $(200 \mathrm{mg} / \mathrm{kg} /$ day $)$ and adrenocorticotropic hormone (ACTH) (60 IU/day IM). Her neurological condition continued to deteriorate. Over the next few months she became increasingly obtunded and required a gastrostomy tube to prevent aspiration.

At age eight months, biopsies were taken of the skin, sural nerve and gastrocnemius muscle. Cryostat sections of the skeletal muscle revealed nonspecific myopathic changes. The most prominent findings included variation in fibre size diameter from 7 to $25 \mu \mathrm{m}$ (normal: $15-18 \mu \mathrm{m}$ ) and a mild increase in endomysial connective tissue. Histochemical analysis was unremarkable. In particular, modified Gomori trichrome staining did not reveal the ragged red fibers typical of many mitochondrial myopathies; moreover, oxidative enzyme studies, including nicotinamide adenine dinucleotide, cytochrome $\mathrm{C}$ oxidase, and succinic acid dehydrogenase did not reveal findings suggestive of a mitochondrial myopathy. Oil red $\mathrm{O}$ staining did not reveal an excess of lipid deposition. Ultrastructural analysis of the skeletal muscle additionally revealed focal but nonspecific A-band loss. Light microscopic evaluation of the peripheral nerve was unremark-able. However, electron microscopy revealed a Schwann cell bearing a single profile suggestive of a mitochondrial paracrystalline inclusion (Figure 1). Electron microscopic evaluation of the skin biopsy was unremarkable.

A portion of the skeletal muscle was sent for quantitative mitochondrial enzyme analysis. This demonstrated a complete absence of Complex I activity. Activity of all other respiratory chain complexes was decreased. When Coenzyme $\mathrm{Q}_{10}$ was

From the Division of Pediatric Neurology, Department of Pediatrics (RJH), Division of Medical Genetics, Department of Pediatrics (EGL), University of Saskatchewan, Saskatoon, SK; Division of Anatomic Pathology (CPD), Children and Women's Health Center of British Columbia, Vancouver, British Columbia, Canada.

Received June 25, 2008. Final Revisions Submitted September 9, 2008. Correspondence to: Richard J. Huntsman, Division of Pediatric Neurology, Department of Pediatrics, Royal University Hospital, 103 Hospital Drive, Saskatoon, Saskatchewan, S7N 0W8, Canada. 


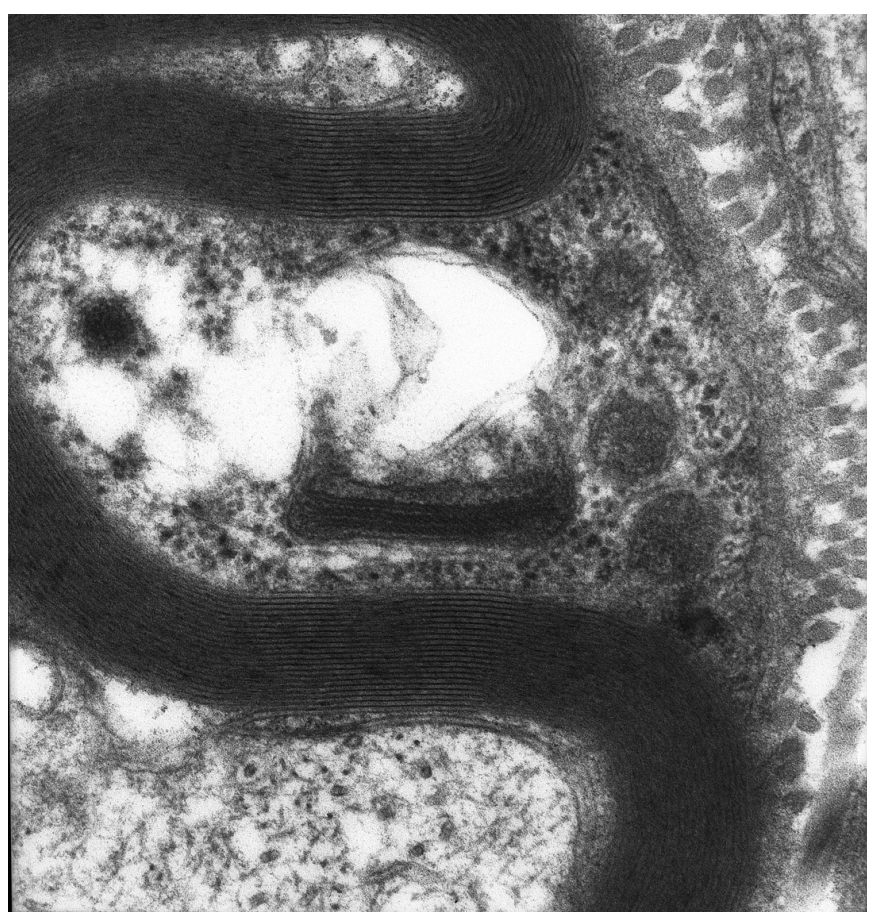

Figure 1: Electron microscopy of the sural nerve demonstrating a profile suggestive of a paracrystalline inclusion (96000X).

added to the muscle, activity of Complex II and III normalized (Decyclubiquinoe ratio). Coenzyme $\mathrm{Q}_{10}$ levels were measured by subjecting lipids extracted from muscle homogenate via reverse phase high performance liquid chromatography. Coenzyme $\mathrm{Q}_{10}$ was completely absent in our patient's muscle sample (Table 1). Western blot analysis revealed the presence of Complexes I, II, III and IV. The results suggested a severe functional disturbance of mitochondrial function secondary to absence of Coenzyme $\mathrm{Q}_{10}$.

Coenzyme $\mathrm{Q}_{10}$ supplementation at $10 \mathrm{mg} / \mathrm{kg} / \mathrm{day}$ was given but has provided no apparent benefit. Initially a powdered preparation was utilized but had to be discontinued until a liquid preparation was available as the powder clogged her gastrostomy tube. Her spasms continued until she was 13 months-of-age despite ongoing treatment with phenobarbital, vigabatrin and Coenzyme $\mathrm{Q}_{10}$ supplementation.

On most recent follow-up at 17 months-of-age she remains profoundly hypotonic and weak. She has no head control. Although she does awaken for brief periods of time, interaction with parents and caregivers is limited to a sporadic smile. Although her spasms have stopped, she continues on vigabatrin and phenobarbital as her EEG remains hypsarrythmic. While she shows no sign of visceral organ dysfunction either clinically or on routine laboratory assessment, she suffers from recurrent episodes of aspiration pneumonia.

Our patient (VII-10) is the youngest of five children born to consanguineous parents (3rd cousins) of First Nations Cree descent (Figure 2). Her parents and four older siblings are healthy. The family history was significant for several distant relatives having an apparent neurodegenerative condition transmitted in an autosomal recessive pattern of inheritance. A paternal cousin of the patient (VII-1) died in infancy with an undiagnosed neurological condition characterized by hypotonia and infantile spasms. This child's parents were second cousins once removed. The father had a paternal cousin that had three children die with a similar condition (V-4 to V-6). An elevation of blood lactate was documented in one of these children. The father had a female paternal second cousin (V-7) that died at age five years of suspected Leigh's disease. Her MRI showed bilaterally increased T2 signal within the basal ganglia. Magnetic Resonance Spectroscopy was suggestive of elevated lactate peaks in the brain parenchyma. There were also two maternal second cousins (a brother and sister) (VI-1 \& VI-2) to the father that died in infancy/early childhood of suspected Leigh's disease. Although these children are not known to be directly related to the mother of the proband, they were all of Cree ancestry.

\section{DiscusSION}

Coenzyme $\mathrm{Q}_{10}$ is a lipophilic compound present in the inner mitochondrial membrane whose primary role is to transfer electrons from complexes I and II to Complex III. Coenzyme $\mathrm{Q}_{10}$ also has other biological roles as an antioxidant and as a regulator of mitochondrial membrane permeability ${ }^{1,2,8}$ Coenzyme $\mathrm{Q}_{10}$ is produced endogenously; nine genes encoded by the nuclear genome are involved in its biosynthesis. To date, mutations in only three of these genes have been found in patients with Coenzyme $\mathrm{Q}_{10}$ deficiency ${ }^{9}$.

Coenzyme $\mathrm{Q}_{10}$ deficiency was first reported in 1989 and since then less than 45 patients have been reported worldwide. Coenzyme $\mathrm{Q}_{10}$ deficiency affects the central nervous system and skeletal muscles predominantly. There are five major phenotypes described, with classification dependent on the relative degree of CNS and muscle involvement. The most common phenotype is the ataxic variant in which the patients present in childhood with

Table: Quantitative mitochondrial enzyme analysis in our patient

\begin{tabular}{l|c|c}
\hline Mitochondrial Enzyme & $\begin{array}{l}\text { Patient Value } \\
(\mu \mathrm{moles} / \mathrm{min} / \mathrm{gm})\end{array}$ & $\begin{array}{l}\text { Control Value } \\
(\mu \mathrm{moles} / \mathrm{min} / \mathrm{gm})\end{array}$ \\
\hline NADH Cytochrome C Reducatase (Complex I) & 0 & $>0.40$ \\
\hline Succinate Dehydrogenase (Complex II) & 0.15 & $>1.30$ \\
\hline $\begin{array}{l}\text { Succinate Cytochrome C Reductase (Complex } \\
\text { II +III) }\end{array}$ & 0.36 & $>0.50$ \\
\hline $\begin{array}{l}\text { Ubiquinone-Ferricytochrme C Oxidoreductase } \\
\text { (Complex III) }\end{array}$ & 0.61 & $>1.71$ \\
\hline $\begin{array}{l}\text { Decyclubiquinone ratio (CoQ10 sensitive } \\
\text { Complex II=III activity) }\end{array}$ & 1.98 & $<3.4$ \\
\hline Cytochrome Oxidase (COX) (Complex IV) & 0.5 & $>1.2$ \\
\hline Citrate Synthase & 6.72 & $6-14$ \\
\hline Coenzyme Q10 ( $\mu \mathrm{g} / \mathrm{g})$ & 0 & $>10$ \\
\hline
\end{tabular}

Control values are provided for comparison. Findings indicate a severe functional disturbance of mitochondrial enzyme function secondary to a deficiency of Coenzyme $\mathrm{Q}_{10}$. 


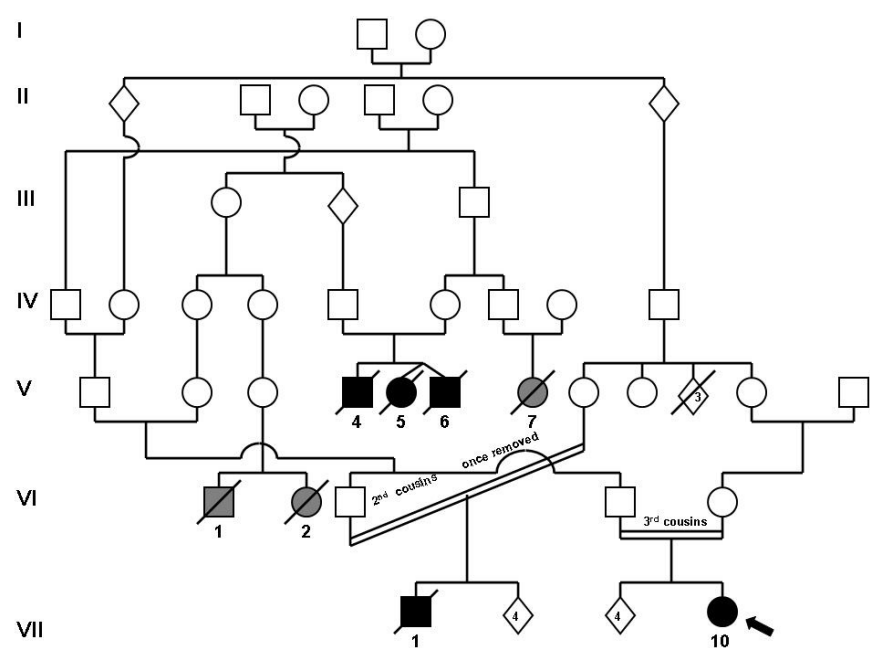

Figure 2: Family pedigree with proband (arrow) and other affected children. Those with infantile spasms and hypotonia are shaded black and those with suspected Leigh's disease are shaded grey. Children that died in early infancy of unknown causes are also identified (nonshaded shape with line). A diamond shape denotes the sex is unknown or has been anonymized to prevent identification of the family. Arabic numbers below the symbols are used to identify individuals in a particular generation (indicated by Roman numerals).

progressive ataxia ${ }^{1,2}$. Neuroimaging studies usually demonstrate cerebellar atrophy. Developmental delay and seizures are not uncommon. Muscle biopsy is usually normal with no stigmata of mitochondrial disease seen.

In the severe infantile multisystemic form onset is usually in the neonatal period ${ }^{1}$. These infants develop severe encephalopathy with hypotonia, seizures, dystonia and ataxia. All have severe renal disease usually in the form of a tubular nephropathy. Lactate levels in blood and CSF are often normal. Neuroimaging usually shows generalized atrophy of the cerebellum and cerebral hemispheres ${ }^{1,5,6}$. The muscle biopsy can be normal or show nonspecific changes such as type $2 \mathrm{~B}$ fiber type atrophy with excess lipid accumulation ${ }^{1,5,6}$.

A Leigh's disease phenotype has also been reported in some infants with Coenzyme $\mathrm{Q}_{10}$ deficiency. These patients all presented in infancy with hypotonia, failure to thrive, and neurodevelopmental regression. All patients had elevated lactate in blood or CSF. Magnetic resonance imaging showed bilateral hyperintense T2 signals within the basal ganglia in keeping with Leigh's disease. Muscle biopsy showed ragged red fibers in only one patient. In two other patients there was only non-specific type 2 muscle fiber atrophy ${ }^{1,3,4}$.

The encephalomyopathic form of Coenzyme $Q_{10}$ deficiency is characterized by progressive proximal muscle weakness, ataxia, and seizures ${ }^{2}$. These patients usually present in early childhood with exercise intolerance and recurrent myoglobinuria. In all patients there was histological evidence of mitochondrial disease on muscle biopsy in the form of ragged red fibers and lipid droplets $^{1,2,10}$.

The myopathic variant is characterized by muscle weakness and exercise intolerance. Onset is usually in preadolescence. Central nervous system involvement (seizures, ataxia) may be present but is mild compared to the myopathy. All patients have elevations of blood and CSF lactate and the muscle biopsies all showed ragged red fibers and lipid droplets ${ }^{1,2}$.

Our patient does not fit any of the above phenotypes, primarily because she had infantile spasms and hysparrythmia on EEG. Unlike infants with the severe multisystemic phenotype, she has yet to show any evidence of visceral organ dysfunction including renal disease. Although her MRI showed cerebral atrophy there was no involvement of the basal ganglia as one would expect to see in patients with Leigh's disease. As well, her blood and CSF lactate levels were normal.

Infantile spasms are an age specific epileptic response of the immature brain to various insults and denote a severe disturbance of CNS function. Mitochondrial diseases were previously felt to be an uncommon cause of infantile spasms. This is partly due to the difficulty in diagnosing some mitochondrial diseases. Normal blood and CSF lactate are not uncommon in children with a mitochondrial disease ${ }^{11}$. Sadleir et al demonstrated that forty percent of children with confirmed or suspected mitochondrial disease had infantile spasms, while conversely eight percent of all children with spasms were shown to have a mitochondrial disease ${ }^{11}$. This makes mitochondrial disease the most important metabolic cause of infantile spasms $^{7,11}$.

Despite Coenzyme $Q_{10}$ supplementation, our patient continued to have infantile spasms and a more general neurological decline. The lack of a clinical response to supplemental Coenzyme $\mathrm{Q}_{10}$ is not unexpected. Oral Coenzyme $\mathrm{Q}_{10}$ has very limited bioavailability due to its hydrophobic nature. While supplemental Coenzyme $\mathrm{Q}_{10}$ has been shown to improve muscle function and normalize metabolic markers such as lactic acidosis, it does not appear to have any effect on CNS symptoms such as seizures and developmental delay. One proposed explanation is that injury to the central nervous system secondary to energy deprivation is irreversible and the ability of Coenzyme $\mathrm{Q}_{10}$ to cross the blood brain barrier is limited ${ }^{2}$. However, it is plausible that supplemental Coenzyme $Q_{10}$ may prevent some of the CNS injuries if given early in the disease course. While Coenzyme $\mathrm{Q}_{10}$ supplementation may be beneficial in patients with Coenzyme $\mathrm{Q}_{10}$ deficiency, it has not been shown to be beneficial in patients with other mitochondrial disorders ${ }^{12}$.

Our patient's muscle biopsy demonstrated non-specific changes with no definite stigmata of mitochondrial disease. The loss of A-bands on electron microscopy can be explained by the use of ACTH to control the spasms. The only feature suggestive of mitochondrial disease was the paracrystalline inclusion body seen in the sural nerve biopsy. The absence of prominent muscle biopsy findings suggestive of a mitochondrial disease in combination with the normal serum and CSF lactate levels could easily have resulted in the diagnosis of coenzyme $\mathrm{Q}_{10}$ deficiency being overlooked in our patient. This raises the distinct possibility that Coenzyme $\mathrm{Q}_{10}$ deficiency has been overlooked in many patients, making it not such a rare disorder.

On reviewing the patient's family history, there appears to be an underlying neurodegenerative condition that is inherited in an 
autosomal recessive pattern. It is very plausible that these children also have Coenzyme $\mathrm{Q}_{10}$ deficiency. If this is the case, we would be able to demonstrate a varied phenotypic expression in the same family and presumably with the same genetic mutation. To date, related children with Coenzyme $\mathrm{Q}_{10}$ deficiency have only been reported to have a similar clinical presentation.

Our patient's case demonstrates that the clinical presentation of primary Coenzyme $\mathrm{Q}_{10}$ deficiency may be more varied than previously thought. A phenotype including infantile spasms, as seen here, has not been previously reported.

Our case also reinforces the point that the absence of an elevated blood or CSF lactate measurement does not exclude a mitochondrial disorder. If one is suspicious that a patient has a mitochondrial disease based on family history, neuroimaging or abnormal lactate measurements, then one should perform quantitative mitochondrial enzyme analysis including a measurement of Coenzyme $\mathrm{Q}_{10}$ even if the muscle histology is normal.

Our case raises the possibility that Coenzyme $\mathrm{Q}_{10}$ deficiency may not be as rare as we had previously thought. The diagnosis is often made only when quantitative enzyme analysis with a measurement of Coenzyme $\mathrm{Q}_{10}$ is performed on a muscle specimen. In many centers MRS is not usually performed in patients with infantile spasms and the paracrystalline inclusion seen on our patient's nerve biopsy could easily have been overlooked. This raises the possibility that other children with cryptogenic infantile spasms, especially those with some degree of muscle weakness, may have a deficiency of Coenzyme $\mathrm{Q}_{10}$.

\section{ACKNOWLEDGEMENTS}

The authors thank Dr. David Price for his assistance in procuring the biopsy specimens.

\section{REFERENCES}

1. Rotig A, Mollet J, Rio M, Munnich A. Infantile and pediatric quinone deficiency diseases. Mitochondrion. 2007;7S:S112-21.

2. Quinzii $C$, DiMauro $S$, Hirano $M$. Human Coenzyme $Q_{10}$ deficiency. Neurochem Res. 2007;32:723-7.

3. Van Maldergem L, Trijbels F, DiMauro S, Sindelar PJ, Musumeci O, Janssen A, et al. Coenzyme Q-responsive Leigh's encephalopathy in two sisters. Ann Neurol. 2002;52:750-4.

4. Lopez LC, Schuelke M, Quinzii CM, Kanki T, Rodenberg RJ, Naini $\mathrm{A}$, et al. Leigh syndrome and nephropathy and $\mathrm{CoQ}_{10}$ deficiency due to decaprenyl diphosphate synthase subunit 2 (PDSS2) mutations. Am J Hum Genet. 2006;79:1125-9.

5. Rahman S, Hargreaves I, Clayton $P$, Heales S. Neonatal presentation of coenzyme $\mathrm{Q}_{10}$ deficiency. J Pediatr. 2001;139: 456-8.

6. Salviati L, Sacconi S, Murer L, Zacchello G, Franceschini L, Laverda AM, et al. Infantile encephalomyopathy and nephropathy with $\mathrm{CoQ}_{10}$ deficiency: $\mathrm{A} \mathrm{CoQ}_{10}$ responsive condition. Neurology. 2005;65:606-8.

7. Shah NS, Mitchell WG, Boles RG. Mitochondrial disorders: A potentially under-recognized etiology of infantile spasms. J Child Neurol. 2002;17:369-72.

8. Carrozzo R, Piemonte F, Tessa F, Lucioli S, Rizza T, Meschini MC, et al. Infantile mitochondrial disorders. Biosci Rep. 2007;27: 105-12.

9. DiMauro S, Quinzii CM, Hirano M. Mutations in coenzyme $\mathrm{Q}_{10}$ biosynthetic genes. J Clin Invest. 2007;117:587-9.

10. Boitier E, Degoul F, Desguerre I, Charpentier C, Francois D, Ponsot $\mathrm{G}$, et al. A case of mitochondrial encephalomyopathy associated with a muscle coenzyme $\mathrm{Q}_{10}$ deficiency. J Neurol Sci. 1998; 156:41-6.

11. Sadleir LG, Connolly MB, Applegarth D, Hendson G, Clarke L, Rakshi C, et al. Spasms in children with definite and probable mitochondrial disease. Eur J Neurol. 2004;11:103-10.

12. McFarland R, Taylor RW, Turnbull DM. Vitamin treatment in mitochondrial cytopathies. In: Baxter P, editor. Vitamin responsive conditions in paediatric neurology. London: Mac Keith Press; 2001.p. 61-77. 